\title{
Environmental and Occupational Exposure
}

National Cancer Institute

\section{Source}

National Cancer Institute. Environmental and Occupational Exposure. NCI Thesaurus.

Code C19447.

The exposure to potentially harmful agents in the environment or to work-related environmental factors. 